\title{
Estereótipos sobre os idosos: o papel da Universidade na redução do ageismo
}

Stereotypes about the elderly: the role of the University in reducing of ageism

El papel de la universidad sobre estereotipos negativos hacia el proceso de envejecimiento Moacir José ROSSINI NETO ${ }^{1}$

Maria Cristina Rosifini ALVES REZENDE ${ }^{\mathbf{1}}$

João Pedro Justino de Oliveira LIMÍRIO ${ }^{1}$

Angelo Camargo DALBEN ${ }^{2}$

Maria Isabel Rosifini Alves REZENDE ${ }^{3}$

Letícia Maria PESCININI-SALZEDAS

Laís Maria PESCININI-E-SALZEDAS ${ }^{4}$

Leda Maria Pescinini SALZEDAS ${ }^{\mathbf{1}}$

UNESP - Univ Estadual Paulista Júlio de Mesquita Filho, Faculdade de Odontologia de Araçatuba 16015-050 Araçatuba- SP, Brasil ${ }^{2}$ Defensoria Pública do Estado de São Paulo, Unidade de Araçatuba 16010-030 Araçatuba- SP, Brasil

${ }^{3}$ Programa de Pós-Graduação em Direito, Faculdade de Direito de Ribeirão Preto da Universidade de São Paulo (USP) 14040-906 Ribeirão Preto - SP, Brasil

${ }^{4}$ Faculdade de Medicina de Marília, Universidade de Marília (UNIMAR) 17525-902 Marília-SP, Brasil

\section{Resumo}

Introdução: O termo ageismo é definido como uma forma de intolerância relacionada com a idade por meio de estereótipos, ou seja, qualquer pessoa poderia ser alvo de discriminação pela idade que tem, sendo os idosos um dos grupos mais vulneráveis. Teoricamente qualquer pessoa pode ser atingida pelo ageísmo ao longo de sua vida, desde que viva o suficiente para envelhecer. Objetivos: O propósito desse trabalho foi apresentar o papel das Universidades no combate ao ageísmo. Métodos: Para a elaboração do presente trabalho as seguintes etapas foram percorridas: estabelecimento da hipótese e objetivos do estudo; estabelecimento de critérios de inclusão e exclusão de artigos (seleção da amostra). Formulou-se a seguinte questão: as Universidades contribuem para a redução do ageísmo? Os artigos foram selecionados utilizando a base de dados The National Library of Medicine, Washington DC (MEDLINE - PubMed) e Google Scholar. As estratégias utilizadas para localizar os artigos tiveram como eixo norteador a pergunta e os critérios de inclusão da revisão, previamente estabelecidos para manter a coerência na busca dos artigos e evitar possíveis vieses. Como descritores foram utilizados os termos "Ageísmo", "Universidades"; Expectativa de Vida" e "Relação entre Gerações", acordando com o Decs. Os critérios de inclusão foram artigos publicados em inglês, espanhol e português com os resumos disponíveis, no período compreendido entre 19902020. A partir da pesquisa preliminar nas bases de dados, leitura do título e resumo, 20 artigos foram selecionados para leitura na íntegra. Resultados: A Universidade, por meio de seus projetos de extensão universitária voltados aos idosos tem contribuído para redução das atitudes que nutrem papéis sociais estereotipados com base na idade das pessoas. Conclusões: As atividades universitárias voltados ao idoso têm contribuído para redução dos estereótipos mantidos pela sociedade e pelos próprios indivíduos com 60 anos ou mais, contribuindo não só para o bem estar e qualidade de vida desse segmento, como também para que a sociedade se beneficie do contato positivo intergeracional.

Descritores: Ageísmo; Resiliência Psicológica; Universidades; Expectativa de Vida; Relação entre Gerações; Qualidade de Vida.

\section{Abstract}

Introduction: The term ageism is defined as a form of age-related intolerance through stereotypes, that is, anyone could be discriminated against by their age, with the elderly being one of the most vulnerable groups. Theoretically anyone can be affected by ageism throughout their life, as long as they live long enough to grow old. Objectives: The purpose of this work was to present the role of Universities in the fight against ageism. Methods: For the elaboration of the present work, the following steps were taken: establishment of the hypothesis and objectives of the study; establishment of inclusion and exclusion criteria for articles (sample selection). The following question was asked: do Universities contribute to the reduction of ageism? The articles were selected using the database The National Library of Medicine, Washington DC (MEDLINE - PubMed) and Google Scholar. The strategies used to locate the articles were guided by the question and the inclusion criteria of the review, previously established to maintain consistency in the search for articles and avoid possible bias. The following descriptors were used: "Ageism", "Universities"; Life Expectancy" and "Intergenerational Relations", agreeing with Decs. The inclusion criteria were articles published in English, Spanish and Portuguese with the available abstracts, in the period between 1990-2020. From the preliminary search in the databases, reading the title and abstract, 20 articles were selected for reading in full. Results: The University, through its university extension projects aimed at the elderly, has contributed to reducing attitudes that nurture stereotyped social roles based on people's age. Conclusions: University activities aimed at the elderly have contributed to reducing stereotypes maintained by society and by individuals aged 60 or over, contributing not only to the well-being and quality of life of this segment, but also for society to benefit from the positive intergenerational contact.

Descriptors: Ageism; Resilience, Psychological; Universities; Life Expectancy; Intergenerational Relations; Quality of Life.

\section{Resumen}

Introducción: El término ageismo se define como una forma de intolerancia relacionada con la edad a través de estereotipos, es decir, cualquier persona podría ser discriminada por su edad, siendo los ancianos uno de los grupos más vulnerables. Teóricamente, cualquier persona puede verse afectada por el ageismo a lo largo de su vida, siempre que viva lo suficiente como para envejecer. Objetivos: El objetivo de este trabajo fue presentar el papel de las universidades en la lucha contra el envejecimiento. Métodos: Para la elaboración de este trabajo, se tomaron los siguientes pasos: establecimiento de la hipótesis y objetivos del estudio; establecimiento de criterios de inclusión y exclusión para artículos (selección de muestra). Se hizo la siguiente pregunta: ¿las universidades contribuyen a la reducción del ageismo? Los artículos fueron seleccionados utilizando la base de datos The National Library of Medicine, Washington DC (MEDLINE - PubMed) y Google Scholar. Las estrategias utilizadas para ubicar los artículos se guiaron por la pregunta y los criterios de inclusión de la revisión, previamente establecidos para mantener la coherencia en la búsqueda de artículos y evitar posibles sesgos. Se utilizaron los siguientes descriptores: "Ageísmo", "Universidades"; Experanza de Vida "y“ Relaciones Intergeneracionales", de acuerdo con Decs. Los criterios de inclusión fueron artículos publicados en inglés, español y portugués con los resúmenes disponibles, entre 1990 y 2020. De la búsqueda preliminar en las bases de datos, la lectura del título y el resumen, se seleccionaron 20 artículos para su lectura completa. Resultados: La Universidad, a través de sus proyectos de extensión universitaria dirigidos a las personas mayores, ha contribuido a reducir las actitudes que nutren los roles sociales estereotipados en función de la edad de las personas. Conclusiones: la actividad universitaria dirigida a las personas mayores han contribuido a reducir los estereotipos mantenidos por la sociedad y por las personas de 60 años o más, contribuyendo no solo al bienestar y la calidad de vida de este segmento, sino también a que la sociedad se beneficie contacto intergeneracional positivo.

Descriptores: Ageísmo; Resiliencia Psicológica; Universidades; Expectativa de Vida; Relaciones Intergeneracionales; Calidad de Vida.

\section{INTRODUÇÃO}

Nos últimos dois séculos a expectativa média de vida dobrou nas populações dos países desenvolvidos ${ }^{1}$. No Brasil, de acordo com os dados da Tábua Completa de Mortalidade para o
Brasil (IBGE) ${ }^{2}$, houve um crescimento de três meses e quatro dias na expectativa média de vida, ao considerarmos o período 2017-2018, passando a ser em média 76,3 anos (72,8 anos para os homens e 79,9 
anos para as mulheres). Aboim ${ }^{3}$ ressalva que a paisagem demográfica das sociedades, em particular das sociedades ocidentais, sofreu grande alteração em razão do envelhecimento acelerado a partir da segunda metade do século $\mathrm{XX}^{1,4}$, para o qual também contribuiu a redução dos índices de mortalidade na infância. O impacto social, econômico, cultural e político do envelhecimento, quando considerada a relação direta entre indivíduo e sociedade, evidencia a urgência da reorganização global frente ao envelhecimento ${ }^{5}$.

Introduzido em 1975 por Buttler ${ }^{6}$, o conceito de ageismo como discriminação ao indivíduo idoso, dentro de um processo de estereotipagem sistemática, pode apresentar, segundo Iversen et al. ${ }^{7}$, contornos negativos ou positivos, implícitos ou explícitos, na esfera micro, meso ou macro. Não raro, em muitas culturas os idosos são estereotipados como indivíduos irritadiços, deprimidos, solitários, pobres, senis, doentes, incapazes de aprender, pouco úteis e sem força de trabalho efetivo ${ }^{8,9}$. Estudos demonstram que os indivíduos expostos a visões negativas do envelhecimento apresentam pior desempenho nas tarefas cognitivas, maior desenvolvimento de problemas de saúde e diminuição da expectativa de vida $^{10-13}$. Meisner ${ }^{14}$ destaca que a adoção generalizada de atitudes negativas em relação ao envelhecimento contribuem para o risco de mortalidade, problemas de saúde funcional e recuperação mais lenta de doenças. Para Gil Barreiro e Trujillo $\mathrm{Gras}^{15}$, os estereótipos negativos em relação ao processo de envelhecimento, mantidos pela sociedade e pelos próprios idosos, são fenômenos que podem sobrecarregar as capacidades adaptativas das pessoas e afetar sua qualidade de vida e saúde.

A Universidade, enquanto instrumento de formação de recursos humanos também responde pela criação das bases para o desenvolvimento e transformação sociocultural. Aliado aos programas de ensino formais, o crescimento dos programas de extensão universitária voltados à população idosa, conhecidos como Universidades Abertas a Terceira Idade, têm contribuído para o ingresso da sociedade no século XXI com um novo cenário de perspectivas para os indivíduos com 60 anos ou mais, contribuindo para seu bem estar e qualidade de vida $^{16}$. O propósito deste trabalho foi promover revisão sistematizada sobre o papel das Universidades na redução dos estereótipos mantidos pela sociedade e pelos próprios indivíduos com 60 anos ou mais.

\section{MATERIAL E MÉTODO}

Para a elaboração do presente trabalho as seguintes etapas foram percorridas: estabelecimento da hipótese e objetivos do estudo; estabelecimento de critérios de inclusão e exclusão de artigos (seleção da amostra). Formulou-se a seguinte questão: as Universidades contribuem para a redução do ageísmo? Os artigos foram selecionados utilizando a base de dados The National Library of Medicine, Washington DC (MEDLINE - PubMed) e Google Scholar. As estratégias utilizadas para localizar os artigos tiveram como eixo norteador a pergunta e os critérios de inclusão da revisão, previamente estabelecidos para manter a coerência na busca dos artigos e evitar possíveis vieses. Como descritores foram utilizados: "Ageísmo", "Resiliência Psicológica", "Universidades"; Expectativa de Vida" e "Relação entre Gerações", acordando com o Decs. Os critérios de inclusão foram artigos publicados em inglês, espanhol e português com os resumos disponíveis, no período compreendido entre 19902020. A partir da pesquisa preliminar nas bases de dados, leitura do título e resumo, 20 artigos foram selecionados para leitura na íntegra (Figura 1).

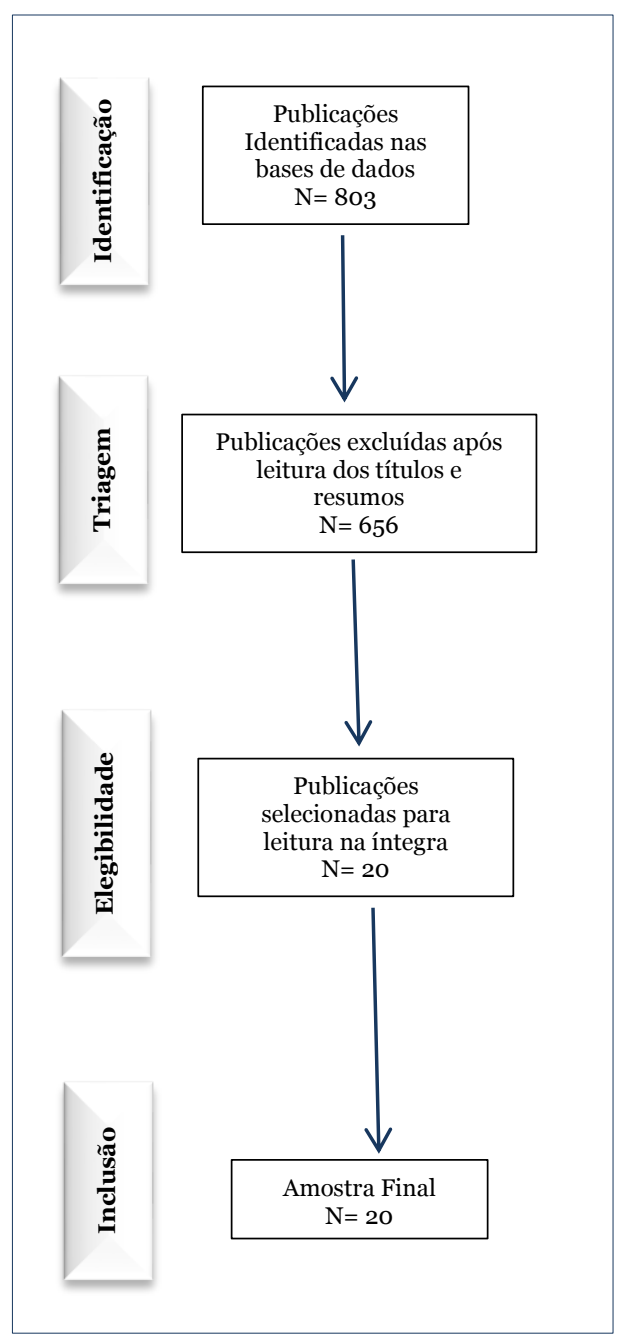

Figura 1: Fluxograma do processo de seleção dos estudos para a revisão.

\section{RESULTADOS E DISCUSSÃO}

Schroyen et al. ${ }^{17}$ estudaram a influência da visão negativa do envelhecimento na relação médicopaciente. Para tanto 40 profissionais de saúde 
(médicos e estudantes de medicina) foram solicitados a gravar um vídeo em que precisavam explicar uma terapia endócrina a dois pacientes fictícios, um com 40 anos e outro com 70 anos de idade. Os resultados mostraram que, quando os participantes explicaram o tratamento ao paciente mais velho, eles usaram expressões mais curtas, fizeram mais repetições, encurtaram enunciados longos, utilizando poucas palavras por minuto. Os efeitos colaterais da terapia, em particular os sexuais, também foram suprimidos no vídeo gravado para o paciente idoso.

Chrisler et al. ${ }^{18}$ estudaram o efeito do ageísmo, sexismo e estereótipos em relação à mulher idosa no sistema de saúde. Para os autores, o ageismo pode resultar no comprometimento da relação médico-paciente, com diálogos infantilizados, além de subestimar relatos de queixas, interpretandoas como "simples sinal de velhice", o que prejudica a adesão das pacientes aos esquemas terapêuticos prescritos e a adoção dos cuidados em saúde.

Arnold et al. ${ }^{19}$ avaliaram a influência de mentores idosos no curso de graduação em Medicina, da Universidade de Missouri (Estados Unidos) como forma de colocar o estudante em contato com idosos saudáveis. Observaram que a abordagem auxiliou os alunos na construção de uma visão multidimensional do envelhecimento, desafiando estereótipos e apreciando a singularidade da vida de seus mentores.

Thornhill et al. ${ }^{20}$, com base no programa de mentores sêniores da Faculdade de Medicina da Universidade da Carolina do Sul (Estados Unidos), acreditam que o acompanhamento de pacientes saudáveis com mais de 65 anos, ao longo de toda a graduação, oferece experiência longitudinal aos graduandos na medida em que permite que os alunos aprendam como seus mentores seniores se comportam no dia-a-dia, avaliando o paciente como um todo: sua saúde, suas necessidades médicas, seus estilos de vida e seus ambientes sociais e físicos.

Bates et al. ${ }^{21}$ ressalvam o papel do Programa de Mentor Sênior da Faculdade de Medicina de Wisconsin (Estados Unidos) como importante estratégia para o aumento de atitudes positivas sobre o envelhecimento e a geriatria .

Levy $^{22}$ atribui à educação de crianças e jovens o fator principal para redução do ageismo. Em 2018 o autor propôs um modelo teórico (PEACE Positive Education about Aging and Contact Experiences) para reduzir o ageismo, com foco em dois fatores promissores: educação sobre 0 envelhecimento e experiências de contato positivas com idosos.

Abrams et al. $^{23}$ examinaram o efeito do contato intergeracional e da ameaça de estereótipo no desempenho cognitivo, na ansiedade, no viés intergrupos e na identificação das pessoas mais velhas. Os autores observaram melhor desempenho em tarefas cognitivas após contato intergeracional positivo. $\mathrm{O}$ efeito moderador foi mediado pela ansiedade relacionada ao experimento. Para os autores, de acordo com a teoria do contato entre grupos, o contato mais positivo foi associado à redução do preconceito e à identificação reduzida de grupos.

Em 2017, Swift et al. ${ }^{12}$ propuseram um modelo de riscos do ageismo para melhor eficiência das políticas públicas voltadas ao envelhecimento ativo, com base na avaliação da modalidade de estereótipo, o processo pelo qual os estereótipos são internalizados e se tornam relevantes; na ameaça de estereótipo, risco percebido de conformidade com estereótipos negativos sobre o grupo de pessoas; e na discriminação do idoso relacionada ao tratamento injusto com base na idade.

A comparação social do idoso a pessoas mais jovens também foi investigada e demonstrou resultar em potencial ameaça baseada em estereótipo ou aumento no desempenho das pessoas mais velhas. Os resultados mostraram que uma comparação ameaçadora pode resultar em desempenho insuficiente por parte de idosos em domínios de tarefas autoestereotipadas de maneira negativa e positiva. $\mathrm{O}$ estudo também demonstrou que a comparação social com pessoas mais jovens pode melhorar o desempenho das pessoas mais velhas em um domínio de tarefas estereotipado positivamente ${ }^{24}$.

Em 2015 Pruchno et $\mathrm{al}^{25}$. integraram as teorias da resiliência ao envelhecimento saudável, segundo as quais os indivíduos que superam as adversidades mantendo altos níveis de desempenho envelhecem de forma saudável. Windle ${ }^{26}$ acrescenta a importância de se considerar a dinâmica da resiliência ao longo da vida, o seu papel no envelhecimento saudável e no gerenciamento de perdas, assim como mudanças no funcionamento cognitivo.

Liébana-Presa et al. $^{27}$ compararam a realidade social por meio da variável coesão, relacionando-a com a resiliência de estudantes universitários de enfermagem do primeiro e terceiro anos dos curso. Seus resultados apontaram que as redes de amizade e apoio dos alunos mostraram alta coesão intrasala de aula, sendo menores no terceiro ano. Não foram encontradas correlações entre coesão e resiliência. Para os autores, a resiliência não parece ser um atributo relacionado à coesão ou vice-versa. No entanto, a alta coesão fornece aos alunos um capital social interessante que pode facilitar a criação de ideias e recursos, além de contribuir para o desempenho acadêmico dentro e fora da sala de aula.

Fernandez-Martinez et al. $^{28}$ analisaram as redes sociais como parâmetro não só para avaliar a relação entre resiliência e engajamento (rendimento acadêmico, dedicação e pró-atividade), como também para aquilatar a importância do uso dessa relação como metodologia educacional capaz de melhorar o 
desempenho acadêmico, criando redes emocionalmente sustentáveis. O estudo, de caráter descritivo foi realizado em 134 estudantes universitários. Os resultados da quantificação do grau de resiliência e engajamento ao curso mostraram valores mais altos no início do ensino universitário. Assim, quanto maior o valor do engajamento, quanto maior o grau de centralidade na rede de amizade; essa relação se inverteu à medida que os estudantes passam para os anos acadêmicos posteriores. Em termos de resiliência, os valores mais altos corresponderam a uma maior centralidade nas redes de amizade. Para os autores, engajamento e resiliência influenciaram as redes de apoio dos estudantes universitários, justificando a implementação de programas que promovam o envolvimento e a resiliência dos estudantes universitários, a fim de melhorar a rede de comunicação.

Para Montepare e Farah $^{29}$ as Universidades Abertas a Terceira Idade, embora se constituam opção educacional desafiadora, oferecem oportunidades de aprendizado ao longo da vida para estudantes de todas as idades por meio das atividades intergeracionais.

Montepare et $\mathrm{al}^{30}$ ressalvam que o envelhecimento populacional mundial em a taxas recordes impõe às instituições universitárias a extensão ao idoso do acesso a várias oportunidades educacionais, proporcionando intercâmbio intergeracional, facilitando o compartilhamento recíproco de conhecimentos entre alunos de diferentes idades e mitigando atitudes negativas frente à diversidade da idade.

Andreoletti e Junho ${ }^{31}$ apontam para a necessidade de esforços na construção de coalizões dentro e fora da universidade que fortaleçam os laços acadêmicos com a comunidade, contribuindo para a diminuição do ageísmo. Chesser e Porter ${ }^{32}$ apontam que as várias estratégias adotadas na Universidade de Manitoba (Canadá) no programa Universidade Aberta a Terceira Idade incentivam a convivência intergeracional, além de promover o diálogo com a comunidade e fomentar a pesquisa interdisciplinar relacionada à idade.

Segundo Clark e Leedahl ${ }^{33}$ os programas de extensão universitários voltados à terceira idade permitem o desenvolvimento de atividades de aprendizagem ao longo da vida, de caráter intergeracional, fornecendo uma base sólida para mudanças no ambiente acadêmico, tornando-o mais "amigável ao envelhecimento".

Leedahl et al.$^{34}$ ressaltam que a aprendizagem intergeracional de serviços no ensino superior afeta positivamente idosos e alunos, assim como corroborado por Andreotti e Howard ${ }^{35}$, que apontaram também a redução dos estereótipos relacionados à idade e aumento da generatividade em adultos mais velhos (redução significativa no envelhecimento na escala de idade de Fraboni para adultos jovens). Os autores acreditam que a aprendizagem intergeracional de serviços, mesmo em pequenas doses, mostra-se promissora para preencher a lacuna geracional ${ }^{35}$.

\section{CONSIDERAÇÕES FINAIS}

O preconceito, a discriminação e o estereótipo com base na idade fundamentam o ageísmo, com impacto altamente negativo sobre a saúde, bem estar e qualidade de vida do idoso. A Universidade, enquanto instrumento de formação de recursos humanos também responde pela criação das bases para o desenvolvimento e transformação sociocultural, na medida em que oferece acesso ao idoso para a participação de atividades intergeracionais. Tais atividades contribuem não só para a superação das adversidades pelo idoso mantendo altos níveis de desempenho e envelhecimento saudável, como também para a redução do ageísmo.

\section{REFERÊNCIAS}

1. Oeppen J, Vaupel JW. Limites quebrados à expectativa de vida. Science. 2002; 296:1029-31.

2. IBGE (Instituto Brasileiro de Geografia e Estatística). Tábuas Completas de Mortalidade. Disponivel em: https://www.ibge.gov.br /estatisticas/sociais/populacao/9126-tabuascompletas-de-mortalidade.html?=\&t=resultados.

3. Aboim S. Narrativas do envelhecimento. Ser velho na sociedade contemporânea. Tempo Social. 2014;26(1):207-32.

4. Vaupel JW, Carey JR, Christensen K, Johnson TE, Yashin AI, Holm NV et al. Biodemographic trajectories of longevity. Science. 1998; 280(5365):855-60.

5. Donizzetti AR. Ageism in an Aging Society: The Role of Knowledge, Anxiety about Aging, and Stereotypes in Young People and Adults. Int J Environ Res Public Health. 2019;16(8):1329.

6. Butler RN. Why Survive? Being Old in America. New York: Harper \& Rowe; 1975.

7. Iversen TN, Larsen L, Solem PE. A conceptual analysis of ageism. Nord Psychol. 2009;61(3):422.

8. Levy BR, Kasl SV, Gill TM. Image of aging scale. Percept Mot Skills. 2004;99(1):208-10.

9. Nelson T. Ageism. In: Nelson T (Ed.), Handbook of prejudice, stereotyping, and discrimination. New York, NY: Psychology Press; 2009. pp-431-40

10. Abrams D, Eller A, Bryant J. An age apart: The effects of intergenerational contact and stereotype threat on performance and intergroup bias. Psychol Aging. 2006;21(4):691-702.

11. Levy BR, Slade MD, Kunkel SR, Kasl SV. Longevity increased by positive self-perceptions of aging. J Pers Soc Psychol. 2002;83(2):261-70. 
12. Swift HJ, Abrams D, Lamont RA, Drury L. The risks of ageism model: how ageism and negative attitudes toward age can be a barrier to active aging. Soc Issues Policy Rev. 2017;11(1):195-231.

13. Wurm S, Diehl M, Kornadt AE, Westerhof GJ, Wahl HW. How do views on aging affect health outcomes in adulthood and late life? Explanations for an established connection. Dev Rev. 2017; 46:27-43.

14. Meisner BA. A meta-analysis of positive and negative age stereotype priming effects on behavior among older adults. J Gerontol B Psychol Sci Soc Sci. 2012;67(1):13-7.

15. Gil Barreiro M, Trujillo Grás O. Estereotipos hacia los ancianos. Rev Cubana Med Gen Integr. 1997;13(1): 34-8.

16. Piato RS, Capalbo LC, Alves Rezende MIR, Lehfeld LS, Alves Rezende MCR. O papel da Universidade Aberta à Terceira Idade na educação ambiental. Arch Health Invest.2014;3(5):66-71.

17. Schroyen S, Adam S, Marquet $M$, et al. Communication of healthcare professionals: Is there ageism? Eur J Cancer Care (Engl). 2018;27(1):10.1111.

18. Chrisler JC, Barney A, Palatino B. Ageism can be hazardous to women's health: ageism, sexism, and stereotypes of older women in the healthcare system. J Soc Issues. 2016;72(1):86-104.

19. Arnold L, Shue CK, Jones D. Implementation of geriatric education into the first and second years of a baccalaureate-MD degree program. Acad Med. 2002;77(9):933-34.

20. Thornhill J 4th, Richeson N, Roberts E. Senior mentor program: a geriatrics focused curriculum. Acad Med. 2002;77(9):934-35.

21. Bates T, Cohan M, Bragg DS, Bedinghaus J. The Medical College of Wisconsin Senior Mentor Program: experience of a lifetime. Gerontol Geriatr Educ. 2006;27(2):93-103.

22. Levy SR. Toward Reducing Ageism: PEACE (Positive Education about Aging and Contact Experiences) Model. Gerontologist. 2018;58(2): 226-32.

23. Abrams D, Eller A, Bryant J. An age apart: the effects of intergenerational contact and stereotype threat on performance and intergroup bias. Psychol Aging. 2006;21(4):691-702.

24. Swift HJ, Abrams D, Marques S. Threat or boost? Social comparison affects older people's performance differently depending on task domain. J Gerontol B Psychol Sci Soc Sci. 2013;68(1):23-30.

25. Pruchno R, Heid AR, Genderson MW. Resilience and successful aging: aligning complementary constructs using a life course approach. Psychol Inquiry. 2015;26(2):200-7.
26. Windle G. The contribution of resilience to healthy ageing. Perspect Public Health. 2012;132(4):159-60.

27. Liébana-Presa C, Andina-Díaz E, Reguera-García MM, Fulgueiras-Carril I, Bermejo-Martínez D, Fernández-Martínez E. Social Network Analysis and Resilience in University Students: An Approach from Cohesiveness. Int J Environ Res Public Health. 2018;15(10):2119.

28. Fernández-Martínez E, Andina-Díaz E, Fernández-Peña R, García-López R, FulgueirasCarril I, Liébana-Presa C. Social Networks, Engagement and Resilience in University Students. Int J Environ Res Public Health. 2017;14(12):1488.

29. Montepare JM, Farah KS. Talk of Ages: Using intergenerational classroom modules to engage older and younger students across the curriculum. Gerontol Geriatr Educ. 2018;39(3):385-94.

30. Montepare JM, Farah KS, Doyle A, Dixon J. Becoming an Age-Friendly University (AFU): Integrating a retirement community on campus. Gerontol Geriatr Educ. 2019; 40(2):179-93.

31. Andreoletti C, June A. Coalition building to create an Age-Friendly University (AFU). Gerontol Geriatr Educ. 2019;40(2):142-52.

32. Chesser S, Porter M. Charting a future for Canada's first Age-Friendly University (AFU). Gerontol Geriatr Educ. 2019;40(2):153-65.

33. Clark PG, Leedahl SN. Becoming and being an Age-Friendly University (AFU): Strategic considerations and practical implications. Gerontol Geriatr Educ. 2019; 40(2):166-78.

34.Leedahl SN, Brasher MS, Estus E, Breck BM, Dennis CB, Clark SC. Implementing an interdisciplinary intergenerational program using the Cyber Seniors ${ }^{\circledR}$ reverse mentoring model within higher education. Gerontol Geriatr Educ. 2018;1-19.

35. Andreoletti C, Howard JL. Bridging the generation gap: Intergenerational service-learning benefits young and old. Gerontol Geriatr Educ. 2018;39(1):46-60.

\section{CONFLITO DE INTERESSES}

Os autores declaram não haver conflitos de interesse.

AUTOR PARA CORRESPONDÊNCIA

Maria Cristina Rosifini Alves Rezende

cristina.rosifini@unesp.br

Submetido em 01/05/2020

Aceito em 02/07/2020 